Swarthmore College

Works

$1-20-1997$

\title{
Blue Light Second Harmonic Generation In The Organic Crystal Ortho-Dicyanovinyl-Anisole
}

Carl H. Grossman

Swarthmore College, cgrossm1@swarthmore.edu

Samuel Aryeh Schulhofer-Wohl , '98

Erik Robert Thoen , '95

Follow this and additional works at: https://works.swarthmore.edu/fac-physics

Part of the Physics Commons

Let us know how access to these works benefits you

\section{Recommended Citation}

Carl H. Grossman; Samuel Aryeh Schulhofer-Wohl , '98; and Erik Robert Thoen , '95. (1997). "Blue Light Second Harmonic Generation In The Organic Crystal Ortho-Dicyanovinyl-Anisole". Applied Physics Letters. Volume 70, Issue 3. 283-285. DOI: 10.1063/1.118393

https://works.swarthmore.edu/fac-physics/89

This work is brought to you for free by Swarthmore College Libraries' Works. It has been accepted for inclusion in Physics \& Astronomy Faculty Works by an authorized administrator of Works. For more information, please contact myworks@swarthmore.edu. 


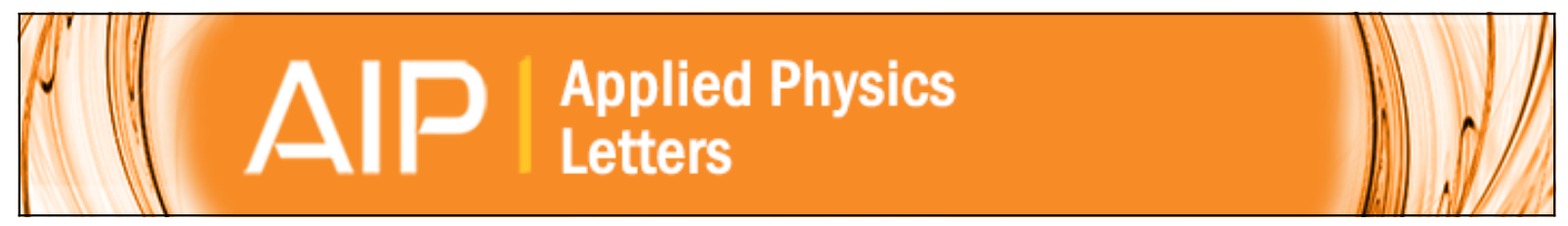

\section{Blue light second harmonic generation in the organic crystal ortho-Dicyanovinyl- anisole}

Carl H. Grossman, Samuel Schulhofer-Wohl, and Erik R. Thoen

Citation: Applied Physics Letters 70, 283 (1997); doi: 10.1063/1.118393

View online: http://dx.doi.org/10.1063/1.118393

View Table of Contents: http://scitation.aip.org/content/aip/journal/apl/70/3?ver=pdfcov

Published by the AIP Publishing

\section{Articles you may be interested in}

Efficient second harmonic generation in a metamaterial with two resonant modes coupled through two varactor diodes

Appl. Phys. Lett. 100, 044103 (2012); 10.1063/1.3679652

Light localization and channeling of atoms in photonic crystals

AIP Conf. Proc. 560, 397 (2001); 10.1063/1.1372737

Blue light emission from an organic nonlinear optical crystal of 4-aminobenzophenone pumped by a laser diode Appl. Phys. Lett. 70, 562 (1997); 10.1063/1.118208

Repetition rate dependence of gray-tracking in KTiOPO 4 during second-harmonic generation at $532 \mathrm{~nm}$ Appl. Phys. Lett. 70, 277 (1997); 10.1063/1.118391

Integrated intracavity quasi-phase-matched second harmonic generation based on periodically poled $\mathrm{Nd}$ :LiTaO 3 Appl. Phys. Lett. 70, 10 (1997); 10.1063/1.119321 


\title{
Blue light second harmonic generation in the organic crystal ortho-Dicyanovinyl-anisole
}

\author{
Carl H. Grossman, Samuel Schulhofer-Wohl, and Erik R. Thoen ${ }^{\text {a) }}$ \\ Department of Physics and Astronomy, Swarthmore College, Swarthmore, Pennsylvania 19081
}

(Received 29 July 1996; accepted for publication 12 November 1996)

Type I phase matched second harmonic generation (SHG) in the organic crystal ortho-Dicyanovinyl-anisole (DIVA) has been measured for a range of near infrared fundamental wavelengths $(855-960 \mathrm{~nm})$. Turning curves for type I phase matched SHG were derived from measured refractive index values and show noncritical phase matching at 860. Measured type I phase matched SHG is in close agreement with the calculated results and gives effective SHG coefficients ranging from 1.9 to 5.9 times as large as $d_{32}$ of potassium niobate. (C) 1997 American Institute of Physics. [S0003-6951(97)00903-0]

Considerable effort is currently centered on designing new organic crystals for efficient bulk second harmonic generation (SHG) and frequency up-conversion of diode laser sources. ${ }^{1-3}$ Molecular second order susceptibilities can be enhanced by designing compounds possessing a large difference between the ground state and first excited state dipole moments, which usually leads to molecular structures having highly polar ground states. ${ }^{4}$ These conditions, together with a desired crystal structure in which the individual molecular axes are partially aligned along a common axis, present a natural dilemma. Ground state dipole-dipole interactions raise the energy of parallel aligned, noncentrosymmetric configurations compared to antiparallel aligned, centrosymmetric configurations. ${ }^{5}$ Such aligned structures have substantial static local fields from the ensemble of ground state dipole moments, often shifting the crystal absorption to lower energies $^{6}$ and reducing the optical transparency range. For example, the polar aromatic compound 2-methyl-4nitroaniline (MNA) possesses a highly aligned polar crystal structure with exceptional second harmonic $^{7}$ and electro-optic ${ }^{8}$ properties. The absorption peak of MNA is significantly shifted from $3.5 \mathrm{eV}$ in solution to $2.9 \mathrm{eV}$ in the crystal, ${ }^{9}$ limiting the optical transparency to wavelengths longer than $500 \mathrm{~nm}$ (absorption coefficient $\alpha<1 \quad \mathrm{~cm}^{-1}$ ).

A compound with a small ground state dipole moment may be more likely to crystallize into a noncentrosymmetric structure with little or no dielectric shift in the excitation energy. If there remains a large difference in dipole moment to an excited state then enhanced SHG properties can result. In other words, this crystal would be robust, have a wider transmission window in the visible and maintain an increased SHG response. Dicyano substituted benzene compounds have been previously studied for their SHG properties $^{10-12}$ and possess a microscopic second order susceptibility comparable to nitroaniline. Crystals of one such compound, 2-dicyanovinylanisole (DIVA), produced an intense SHG from a fundamental at $1064 \mathrm{~nm} .{ }^{13}$ Importantly, though, DIVA molecules have a moderate ground state dipole moment $(\approx 6.5$ debye $)$ and easily form high quality, stable crystals. The absorption edge is only slightly red-

\footnotetext{
a) Department of Electrical Engineering and Computer Science and Research Laboratory of Electronics, Massachusetts Institute of Technology, Cambridge, MA 02139.
}

shifted to about $440 \mathrm{~nm}\left(\alpha<1 \mathrm{~cm}^{-1}\right)$ due to the low degree of static dipolar fields.

Here we present measured type I phase matched SHG data from DIVA crystals and the corresponding tuning curve. Samples of DIVA crystals were grown from vapor generated by vacuum sublimation to produce long columnar crystals with highly flat and parallel facets. The crystal unit cell structure is monoclinic with space group symmetry $P 2_{1}$ and unit cell parameters $\mathrm{a}=11.532, \mathrm{~b}=5.460, \mathrm{c}=7.920 \AA, \alpha=\gamma$ $=90^{\circ}, \beta=97.83^{\circ} .{ }^{13}$ Figure 1 shows the molecular orientation in the unit cell, its relation to the external morphology, and principal dielectric coordinates. DIVA crystals are optically biaxial $\left(2 \mathrm{~V}=70 \pm 2^{\circ}\right)$ with principal dielectric $y$ axis fixed to the crystallographic $\mathrm{b}$ axis and principal $x$ and $z$ axes in the $\mathrm{a}-\mathrm{c}$ plane. The principal $z$-axis orientation was measured with a polarizing microscope at $15^{\circ}$ from the (001) face normal. In Fig. 1 the vinyl bonds and one of the benzene ring axes are aligned centrosymmetrically along the $z$ axis. The large polar response along the $z$ axis (refractive index of $1.8-2.0)$ is most likely due to this alignment. The other benzene ring axes are tilted about $50^{\circ}$ in a "herringbone" pattern about the $y$ axis. This structure is responsible for the large second order polarizability along the $y$ axis for incident

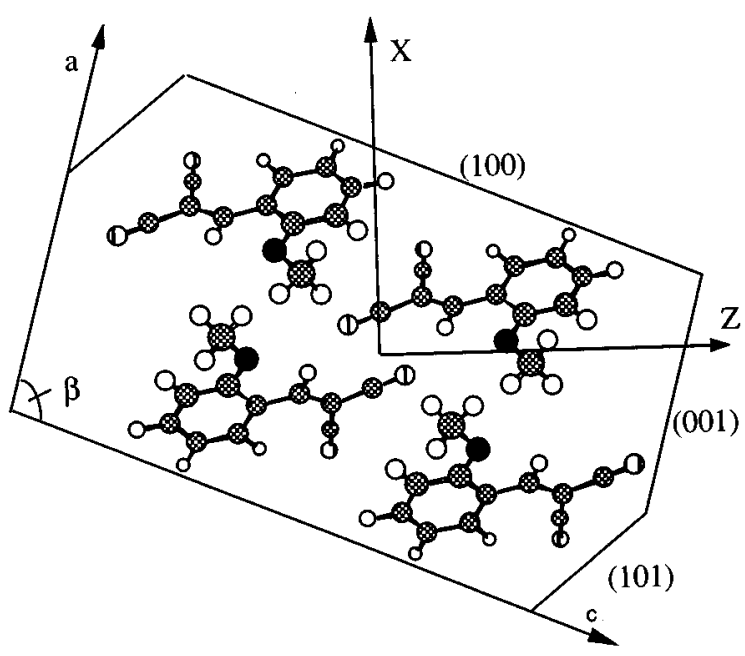

FIG. 1. Crystal unit cell of Dicyanovinylanisole (DIVA) (010) projection. Crystallographic $a$ and $c$ axes form an angle of $97.83^{\circ}$ and the principal dielectric $X$ and $Z$ axes are shown in the measured orientation. 
TABLE I. Sellmeier coefficients for the principal refractive index values of DIVA according to the relation $n^{2}=A+B \lambda^{2} /\left(\lambda_{0}^{2}-\lambda^{2}\right)$.

\begin{tabular}{clll}
\hline \hline Principal axis & \multicolumn{1}{c}{$\mathrm{A}$} & \multicolumn{1}{c}{$\mathrm{B}$} & \multicolumn{1}{c}{$\lambda_{0}$} \\
\hline$x$ & 0.61726 & 1.7027 & 0.14562 \\
$y$ & 2.4254 & 0.18362 & 0.39290 \\
$z$ & 2.7008 & 0.66023 & 0.39536 \\
\hline \hline
\end{tabular}

fields polarized along the $y$ and $z$ axes, resulting in large $d_{23}$ and $d_{25}$ SHG coefficients and a substantial $d_{22}$ coefficient. $^{5}$ (The SHG coefficients for the $P 2_{1}$ symmetry class are $d_{14}, d_{16}, d_{21}, d_{22}, d_{23}, d_{25}, d_{34}$, and $d_{36}$.) The experimental configuration described below couples the terms $d_{21}, d_{23}$, and $d_{25}$ to produce SHG along the $y$ axis.

Refractive index values were determined at several laser wavelengths from the angle dependent reflectivity of an oriented crystal. Results were used to fit an approximate Sellmeier dispersion relation for each of the principal dielectric directions. The best fit Sellmeier coefficients are given in Table I and corresponding plots are shown in Fig. 2. The Sellmeier coefficients $\lambda_{0}$ give a rough estimate of the molecular absorption peak in the crystalline environment of 394 $\mathrm{nm}$. As expected, this is only slightly redshifted from the absorption peak in solution of $365 \mathrm{~nm}$.

Phase matched blue-green SHG was measured for 18 fundamental wavelengths in the range 855-1064 $\mathrm{nm}$. The source of fundamental laser radiation was a pulsed, tunable Ti:sapphire laser end pumped by a frequency doubled $\mathrm{Nd}$ :YAG laser and the direct output of a $\mathrm{Nd}$ :YAG laser. Except for the laser source, the experimental setup was

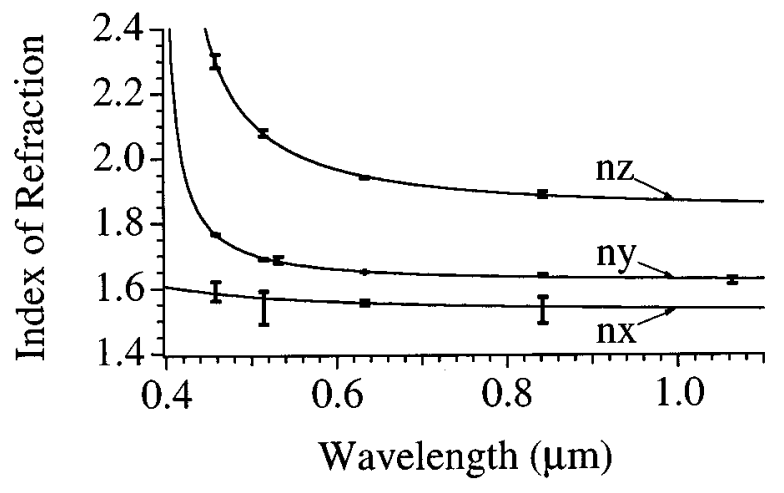

FIG. 2. Refractive index values and Sellmeier Plots for the principal $x, y$, and $z$ axes of DIVA crystals.

nearly identical to that in previously published work. ${ }^{14}$ The sample was rotated about the principal $y$ axis with a stepper motor controlled stage. Maker Fringe SHG and phase matched SHG patterns were measured as a function of stage rotation angle. The fundamental beam was incident to the (100) face, propagating and polarized in the principal $x-z$ plane. Each sample data set was calibrated against a $\mathrm{KNbO}_{3}$ reference SHG measurement subsequently taken under similar conditions. The only difference between the sample and reference SHG runs was the combination of calibrated optical density filters at the input face of the photomultiplier tube's (PMT) photocathode.

Each data set was fit to the Maker Fringe function relating the SHG power to the angle of incidence ${ }^{14}$

$$
\begin{aligned}
P_{2 \omega}= & \left\{\frac{512 \pi^{3}}{A} d_{\mathrm{eff}}^{2} P_{\omega}^{2}\right\} t_{\omega}^{4} 2 n_{2 \omega} \cos \theta_{2 \omega}^{\prime} \frac{\left(\cos \theta+n_{\omega} \cos \theta_{\omega}^{\prime}\right)\left(n_{\omega} \cos \theta_{\omega}^{\prime}+n_{2 \omega} \cos \theta_{2 \omega}^{\prime}\right)}{\left(n_{2 \omega}^{2}-n_{\omega}^{2}\right)\left(n_{2 \omega} \cos \theta_{2 \omega}^{\prime}+\cos \theta\right)^{3}} \\
& \times \sin ^{2}\left[\frac{2 \pi L}{\lambda}\left(n_{2 \omega} \cos \theta_{2 \omega}^{\prime}-n_{\omega} \cos \theta_{\omega}^{\prime}\right)\right],
\end{aligned}
$$

where $n_{\omega}$ and $n_{2 \omega}$ are the refractive index at $\omega$ and $2 \omega, \theta$ is the angle of incidence, $\theta_{\omega}^{\prime}$ and $\theta_{2 \omega}^{\prime}$ are the angles of refraction given by Snell's Law at $\omega$ and $2 \omega, d_{\text {eff }}$ is the effective SHG coefficient, $P_{\omega}$ is the incident fundamental power, $t_{\omega}$ is the fundamental field transmission factor at the crystal input face, $L$ is the crystal length, $A$ is the beam cross sectional area, and $\lambda$ is the fundamental wavelength. A typical data set and its best fit to Eq. (1) are shown in Fig. 3. In the case of the $\mathrm{KNbO}_{3}$ reference, data was analyzed from Eq. (1) with the term $d_{\text {eff }}$ replaced with $d_{32}\left[\left(d_{31} / d_{32}\right) \cos ^{2} \theta_{x}+\cos ^{2} \theta_{y}\right]$, where the angles $\theta_{x}$ and $\theta_{y}$ are between $\mathbf{E}_{\omega}$ and the principal $x$ and $y$ axes, respectively. ${ }^{15}$ The effective SHG coefficient was calculated according to

$$
d_{\text {eff }}=d_{32}\left(\mathrm{KNbO}_{3}\right) \cdot \sqrt{\frac{A_{\text {Sample }}}{A_{\text {reference }}}} .
$$

As seen in Fig. $4, d_{\text {eff }}$ ranges from 1.9 to $5.9( \pm 2 \%)$ in units of $d_{32}$ of $\mathrm{KNbO}_{3}$. The considerable increase in effective co-

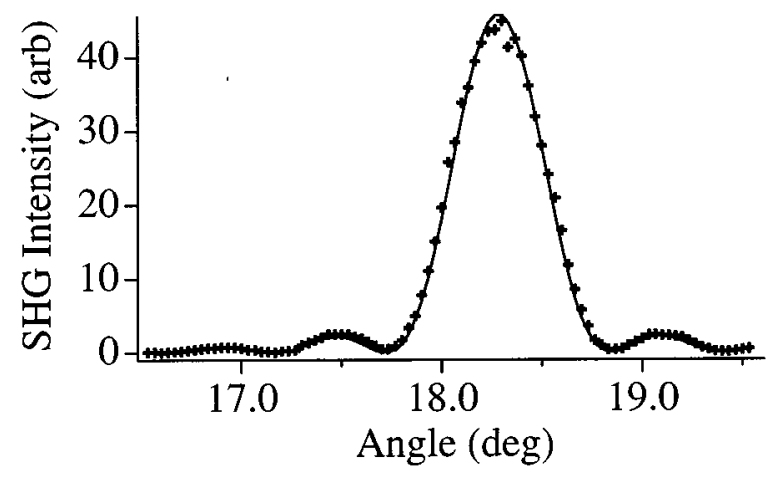

FIG. 3. SHG as a function of incident external angle around the phase matched SHG angle at $\lambda=948.5 \mathrm{~nm}$. The crosses are measured data and the solid line is the best fit to Eq. (1). 


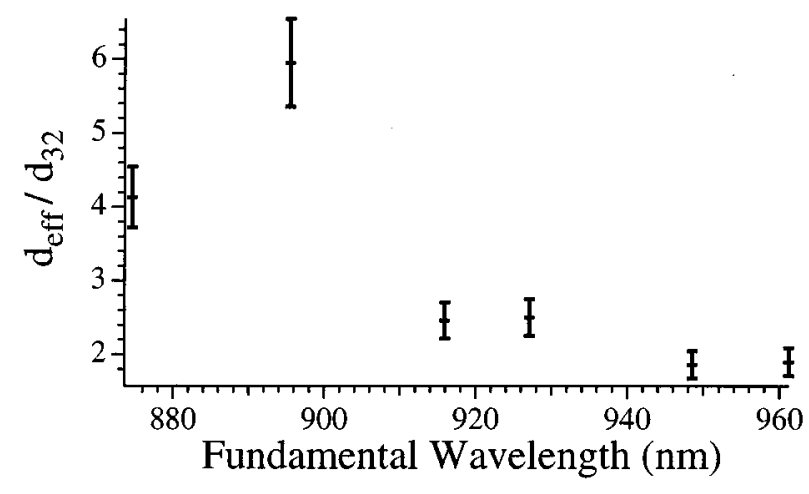

FIG. 4. Effective coefficient for phase matched SHG in DIVA. The reference was $\mathrm{KNbO}_{2}$ which has a reported value for $d_{32}$ of $18.3 \mathrm{pm} / \mathrm{V}$ at 1.064 $\mu \mathrm{m}$.

efficient at shorter wavelengths can be attributed to resonance enhancement and a more favorable molecular orientation. As described earlier, the molecular positions in the crystalline lattice render the coefficient $d_{23}$ larger than $d_{21}$. Phase matching peaks at the shorter wavelengths occur with smaller $\theta_{z}$ value, thus increasing the contribution from the larger $d_{23}$ term. In addition, the SHG coefficients increase for second harmonic photon energies approaching the transition energy of the first dipole allowed excited state. ${ }^{16}$

Calculations of the phase matched SHG in DIVA crystals were based on the measured refractive index values and the fitted Sellmeier coefficients. For SHG polarized along the principal $y$ axis and a fundamental polarized in the $x-z$ plane, type I phase-matching occurs when

$$
\sin ^{2} \theta_{\mathrm{PM}}=n_{z, \omega}^{2} \frac{\left(1-n_{x, \omega}^{2} / n_{y, 2 \omega}^{2}\right)}{\left(n_{z, \omega}^{2}-n_{x, \omega}^{2}\right)},
$$

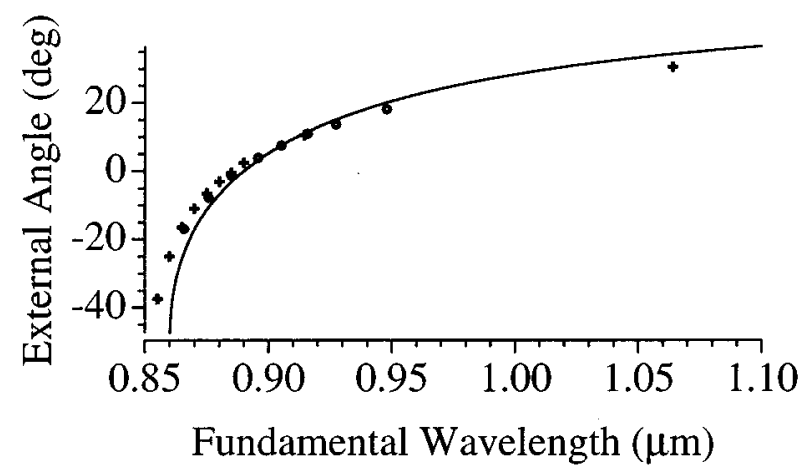

FIG. 5. Tuning curve for type I phase matched SHG in DIVA. The circles and crosses are points measured from SHG experiments and the solid line is determined from the refractive index values and their dispersion. The circles and crosses correspond to different mirror sets in the Ti:sapphire laser except for the data at $1064 \mathrm{~nm}$, which is from the direct output of the YAG laser. where $\theta_{\mathrm{PM}}$ is the internal polar population angle of the beam in the principal coordinate system. The dependence of $\theta_{\mathrm{PM}}$ on fundamental wavelength was calculated and converted into the external angles of the (100) crystal face. As shown in Fig. 5, the calculated tuning curve is in good agreement with the measured angles of peak phase matched SHG in the fundamental wavelength range of $855-960 \mathrm{~nm}$. The type I phase matched SHG tuning curve shows noncritical phase matching at $860 \mathrm{~nm}$. However, due to an absorption band edge at $440 \mathrm{~nm}$, the noncritical type I phase matched SHG is largely absorbed $\left(\alpha \approx 7.5 \mathrm{~cm}^{-1}\right)$.

In conclusion, the linear optical properties of DIVA were measured and used to calculate type I phase matching conditions. Good agreement between calculated and measured type I phase matching directions has been demonstrated for fundamental wavelengths ranging from 855 to $960 \mathrm{~nm}$. A large effective nonlinear optical coefficient for type I phase matched SHG was measured in the range diode laser sources.

Acknowledgment is made to the Research Corporation and the Donors of the Petroleum Research Fund, administered by the American Chemical Society, for partial support of this research. The authors would like to thank Tatsuo Wada, Hiroyuki Sasabe, and Shinji Yamada for their valuable help in the synthesis of DIVA compound and to Geoffrey Park for his help in assembling the experiment and in computer analysis.

${ }^{1}$ See, for example, D. S. Chemla and J. Zyss, Nonlinear Optical Properties of Organic Molecules and Crystals (Academic, Orlando, 1987), Vols. 1 and 2.

${ }^{2}$ M. Kitazawa, R. Higuchi, M. Takahashi, T. Wada, and H. Sasabe, J. Appl. Phys. 78, 709 (1995).

${ }^{3}$ J. Kawamata, K. Inoue, and T. Inabe, Appl. Phys. Lett. 66, 3102 (1995).

${ }^{4}$ S. J. Lalama and A. F. Garito, Phys. Rev. A 20, 1179 (1979).

${ }^{5}$ J. Zyss, D. S. Chemla, and J. F. Nicoud, J. Chem. Phys. 74, 4800 (1981).

${ }^{6}$ C. W. Dirk, R. J. Twieg, and G. Wagnière, J. Am. Chem. Soc. 108, 5387 (1986).

${ }^{7}$ B. F. Levine, C. G. Bethea, C. D. Thurmond, R. T. Lynch, and J. L. Bernstein, J. Appl. Phys. 50, 2523 (1979).

${ }^{8}$ G. F. Lipscomb, A. F. Garito, and R. S. Narang, J. Chem. Phys. 75, 1509 (1981).

${ }^{9}$ Y. Tokura, A. Kurita, and T. Koda, Phys. Rev. B 31, 2588 (1985).

${ }^{10}$ Y. Mori, M. Okamoto, T. Wada, and H. Sasabe, Mater. Res. Soc. Symp. Proc. 109, 345 (1988)

${ }^{11}$ H. E. Katz, K. D. Singer, J. E. Sohn, C. W. Dirk, L. A. King, and H. M. Gordon, J. Am. Chem. Soc. 109, 6561 (1987).

${ }^{12}$ J. B. Stamoff, A. Buckley, G. Calundann, E. W. Choe, R. DiMartino, G. Khanarian, T. Leslie, G. Nelson, S. Steutz, C. C. Teng, and Y. H. Yoon, Proc. SPIE 682, 85 (1986).

${ }^{13}$ C. Grossman, T. Wada, S. Yamada, A. Yamada, H. Sasabe, and A. F. Garito, Springer Proc. Phys. 36, 214 (1989).

${ }^{14}$ J. Jerphagnon and S. K. Kurtz, J. Appl. Phys. 41, 1667 (1970).

${ }^{15}$ Y. Q. Yao and T. S. Fahlen, J. Appl. Phys. 55, 65 (1984).

${ }^{16}$ C. H. Grossman, J. R. Heflin, K. Y. Wong, O. Zamani-Khamiri, and A. F. Garito, in Nonlinear Optical Effects in Organic Polymers, NATO-ASI Series E, Vol. 162, edited by J. Messier, F. Kajzar, P. Prasad, and D. Ulrich (Kluwer Academic, Boston, 1989), pp. 61-78. 